\title{
Telangiectasia, CTCAE 5.0
}

National Cancer Institute

\section{Source}

National Cancer Institute. Telangiectasia, CT CAE 5.0. NCI Thesaurus. Code C146740.

A disorder characterized by local dilatation of small vessels resulting in red discoloration of the skin or mucous membranes. 